\title{
ANÁLISE DOS \\ RESULTADOS \\ DO PRÉ-TESTE DA \\ PROVINHA BRASIL \\ DE MATEMÁTICA
}

MARCELO CÂMARA

\section{RESUMO}

Nesse trabalho, apresentamos uma análise do rendimento de alunos de segundo ano de escolaridade na resolução de problemas de estrutura aditiva com base nos resultados da pré-testagem dos itens que comporão a Provinha Brasil de Matemática. Foram aplicados 192 itens a 12 mil alunos de diferentes unidades da Federação. Realizamos a análise considerando algumas variáveis presentes nos problemas, tais como o tipo de contexto, a presença ou não de imagens, a magnitude dos números envolvidos e a localização dos dados no problema. Os resultados mostram que, em função da estrutura do problema, a alteração dessas variáveis provoca diferenças no índice de sucesso dos alunos.

PALAVRAS-CHAVE PROVINHA BRASIL DE MATEMÁTICA • RENDIMENTO ESCOLAR • RESOLUÇÃO DE PROBLEMAS • AVALIAÇÃO DA APRENDIZAGEM. 


\section{RESUMEN}

En este trabajo presentamos un análisis del rendimiento de los alumnos del segundo año de la escuela primaria en la resolución de resolver los problemas de estructura aditiva, basada en los resultados de la pre-evaluación previa de los items que componen la "Provinha Brasil de Matemática". Se aplicaron de 192 ítems a 12 mil alumnos de diferentes escuelas de Brasil. Realizamos el análisis considerando algunas de las variables presentes en los problemas, tales como el tipo de contexto, la presencia o ausencia de imágenes, la magnitud de los números implicados y la ubicación de los datos en el problema. Los resultados muestran que dependiendo de la estructura del problema, la alteración de esas variables produce diferencias en el índice de éxito de los alumnos.

PALABRAS CLAVES PROVINHA BRASIL DE MATEMÁTICA •

RENDIMIENTO DEL ALUNO • SOLUCIÓN DE PROBLEMAS •

EVALUACIÓN DE LA APRENDIZAJE.

\section{ABSTRACT}

This study presents an analysis of student performance in the second year of elementary school trying to solve problems in additive structure. The analysis is based on the results collected from the pre-test of the items that will make up the "Provinha Brasil de Matemática" (Brazilian Math test). A total of 192 items were submitted to 12 thousand students from different units of the Federation. The analysis took into consideration some of the variables from those items, such as type of context, the presence or absence of images, the magnitude of the numbers involved, and the location of the data in the problem. The results show that, depending on the structure of the problem, changing these variables brings about differences in the success rate of students.

KEYWORDS PROVINHA BRASIL DE MATEMÁTICA (BRAZILIAN MATH TEST) • STUDENT ACHIEVEMENT • PROBLEM SOLVING • LEARNING EVALUATION. 


\section{INTRODUÇÃO}

A discussão sobre avaliação consolidou-se nas duas últimas décadas, no Brasil, particularmente quando se trata de avaliar sistemas de ensino e materiais didáticos. Hoje, temos diferentes sistemáticas de avaliação que são aplicadas nos diversos níveis deensino. Na busca de informações que permitam a elaboração de políticas públicas que levem à melhoria da qualidade da educação, as avaliações em larga escala têm sido implementadas não somente pelo governo federal, mas, também, pelos governos estaduais e municipais.

As avaliações em larga escala podem apresentar resultados bastante úteis para o processo de ensino e aprendizagem, "desde que não se limitem a apenas indicar diagnósticos simplistas, tais como a situação está ruim, melhorou ou piorou" (CÂMARA; MELO, 2009). O mais importante, nesse tipo de avaliação, é possibilitar ao professor que tenha "acesso ao que o aluno está mostrando como conhecimento construído, por meio das estratégias que ele adota no processo de resolução de problemas” (ARAUJO; CÂMARA, 2009).

Entretanto, quando se pensa na avaliação em sala de aula, aquela que interessa diretamente ao professor, pouco se tem avançado. Ainda hoje, prevalecem os aspectos da avaliação ligados a 
diferentes ideologias, mas que não se articulam com os conhecimentos que cabe à escola fazer com que seus alunos adquiram. Isso se reflete sobremaneira na formação dos professores, que ficam privados de melhor formação no que se refere às questões da avaliação.

A consequência desse fenômeno aparece de maneira inequívoca nas práticas de sala de aula. Na escola, a avaliação ainda é percebida como um ato burocrático, como uma "prática institucional que responde unicamente à necessidade de controle que a instituição tem sobre os atores do sistema de ensino" (CÂMARA, 2000), o professor e seus alunos.

Se pensarmos na comunidade dos professores de matemática, no que tange à questão da avaliação, o que se encontra é um sentimento de mal-estar, uma sensação de desconfiança. Segundo Câmara (2000),

[...] podemos dizer que a avaliação escolar se realiza em paralelo ao corpo docente; a interpretação dos resultados de uma avaliação, tão carregada de consequências, não é muito bem reconhecida por esse mesmo corpo. (p. 124)

A ênfase no aspecto burocrático da avaliação faz com que seu instrumento de coleta de informações, privilegiado pela quase totalidade dos professores, a prova escrita, não forneça elementos que permitam ao professor adotar estratégias didáticas mais eficientes. Câmara (2009) mostra que, muitas vezes, os resultados de uma prova escrita mascaram as aprendizagens realizadas pelos alunos.

\section{A PROVINHA BRASIL}

Na busca de superar essas limitações, o Ministério da Educação, por meio do Instituto Nacional de Estudos e Pesquisas Educacionais Anísio Teixeira (Inep), lança, em 2008, a Provinha Brasil de Língua Portuguesa. Trata-se de uma prova aplicada a alunos ao final de seu segundo ano de escolarização que objetiva oferecer ao professor instrumentos para que possa identificar, de forma efetiva, como se encontra o desenvolvimento do processo de alfabetização em língua materna de seus alunos. 
Ao contrário das avaliações em larga escala habituais, a Provinha Brasil de Língua Portuguesa tem seus resultados analisados pelo professor em sua sala de aula. Inclusive, os materiais oferecidos ao professor, que acompanham a prova propriamente dita, apontam as possíveis dificuldades dos alunos e possíveis estratégias didáticas que permitam superar essas dificuldades.

É também em 2008 que o Inep começa a preparar a Provinha Brasil de Matemática (PBM). No mesmo modelo da Provinha de Língua Portuguesa, a PBM se propõe a contribuir para que o professor possa identificar o nível de alfabetização matemática de seus alunos. Para isso, foi elaborada uma matriz de descritores de avaliação contemplando seis competências: compreensão dos números naturais, resolução de problemas envolvendo adição e subtração, resolução de problemas com as ideias de multiplicação e divisão, reconhecimento de representações de figuras geométricas planas e espaciais, identificação e comparação de algumas grandezas e, por fim, leitura e interpretação de tabelas e gráficos de colunas.

Com base nessa matriz foram elaborados 900 itens, dos quais 192 foram pré-testados ao final de 2010. Essa pré-testagem foi realizada com aproximadamente 12 mil alunos de 335 escolas de 11 estados da Federação. Após o tratamento das respostas dos alunos por meio da Teoria da Resposta ao Item (TRI), foram excluídos 60 itens que não responderam aos padrões estatísticos pré-definidos $(\mathrm{c}<0,25$ e $0,58<\mathrm{a}<1,80)$. O resultado da pré-testagem permitiu a elaboração de uma escala de proficiência e, baseada nela, foram elaborados os materiais que compõem o kit para o professor, incluindo a prova relativa ao segundo semestre de 2011.

No presente trabalho, buscaremos realizar uma análise dos resultados da pré-testagem da PBM. Em particular, nosso objetivo foi centrado nas respostas dos alunos aos itens associados aos dois descritores relacionados às ideias de adição e de subtração.

\section{ADIÇÃO E SUBTRAÇÃO}

São inúmeros os trabalhos que investigam as produções dos alunos na resolução de problemas envolvendo adição e subtração, considerado o campo conceitual das estruturas aditivas 
(VERGNAUD, 1990). Grande parte desses estudos investiga, do ponto de vista da cognição, como a criança se comporta no processo de resolução de problemas desse campo conceitual. Isso levou à criação de diversos modelos teóricos para classificar as diferentes estruturas aditivas; o mais explorado dentre eles talvez seja a classificação apresentada por Vergnaud (1982). Esse modelo deu origem a diversos outros, como, por exemplo, o adotado pelos Parâmetros Curriculares Nacionais - PCN - (BRASIL, 1997).

Em nosso trabalho, não pretendemos investigar a performance dos alunos em função da estrutura do problema (embora não seja possível nos furtarmos a alguns comentários), mas sim identificar como a alteração de algumas variáveis nos problemas de adição e subtração podem (ou não) modificar o desempenho dos sujeitos.

Por isso, adotamos a classificação proposta pela matriz de referência de avaliação da Provinha Brasil de Matemática. Nessa matriz, os dois descritores relativos à resolução de problemas envolvendo adição e subtração indicam as ideias de juntar, acrescentar, retirar, completar e comparar quantidades. Por questões de limitação de espaço no texto, somente apresentamos exemplos de problemas envolvendo essas ideias:

Juntar: João tem 5 figurinhas e Maria tem 3. Quantas figurinhas os dois têm, juntos?

Acrescentar: João tinha 5 figurinhas e comprou mais 3 . Quantas figurinhas João tem agora?

Retirar: João tinha 5 figurinhas e deu 3 para Maria. Com quantas figurinhas João ficou?

Completar: João tem 5 figurinhas e Maria tem 3. Quantas figurinhas Maria deve ganhar para ficar com a mesma quantidade de João?

Comparar: João tem 5 figurinhas e Maria tem 3. Quantas figurinhas João tem a mais que Maria?

\section{MÉTODO}

As cinco ideias apresentadas anteriormente são agrupadas em dois descritores da matriz de referência. As três primeiras aparecem no descritor D2.1; e nas recomendações para os elaboradores de itens é indicado que os problemas podem ter ou não imagens, 
que devem ser contextualizados e que, em relação à magnitude dos números envolvidos, as parcelas (no caso da adição) e minuendo (no caso da subtração) não podem exceder 20 unidades. Já os problemas envolvendo as ideias de completar e comparar, contempladas no descritor D2.2, além de serem contextualizados, devem apresentar imagens como suporte; e a magnitude dos números envolvidos não deve exceder 20 unidades.

Na medida em que os itens pertencem ao banco de itens do Inep, eles não serão abertos nesse trabalho. Quando necessário, buscaremos fornecer ao leitor uma ideia sobre a estrutura e outras variáveis presentes no item.

Nossa análise recai sobre um total de 25 itens, sendo 14 relativos ao descritor D2.1 e 11 relativos ao descritor D2.2. Além de identificar o rendimento dos alunos para cada ideia envolvida no problema, adotamos como categorias de análise o contexto do problema, a presença ou não de imagens (no caso do descritor D2.1), a magnitude dos valores envolvidos e a localização dos dados necessários à sua resolução (imagem e/ou enunciado).

É importante ressaltar que se trata de uma análise do rendimento dos alunos em função de variáveis presentes nos itens; portanto, de um estudo diagnóstico. Isso significa dizer que não teremos a preocupação de articular os resultados com os aportes teóricos da área de educação matemática, o que não seria viável em função das limitações próprias a esse tipo de texto.

\section{ANÁLISE}

A análise será realizada em dois momentos. No primeiro, trataremos dos 14 itens relativos às ideias de juntar, acrescentar e retirar, referentes ao descritor D2.1. No segundo momento, vamos analisar os itens que tratam das ideias de completar e comparar, associados ao descritor D2.2.

Para facilitar a compreensão, arredondamos os percentuais para números inteiros, apesar dos resultados da TRI serem apresentados com duas casas decimais. É preciso ressaltar também que, como tivemos a oportunidade de acompanhar a aplicação da pré-testagem em algumas escolas (com a realização de 
registros), eventualmente iremos recuperar informações que possam esclarecer certos resultados.

Antes de iniciar a análise, vamos situar nosso objeto (problemas envolvendo adição e subtração) nos resultados totais, para todas as competências exploradas nas provas. Esses resultados são mostrados na tabela a seguir.

TABELA 1 - Rendimento quanto à habilidade e bloco de competência

\begin{tabular}{|c|c|c|c|}
\hline COMPETÊNCIA & HABILIDADE & \multicolumn{2}{|c|}{$\%$ DE ACERTO } \\
\hline \multirow{5}{*}{ Números } & Contar elementos & 95 & \multirow{5}{*}{87} \\
\hline & Reconhecer numerais & 82 & \\
\hline & Comparar quantidades & 86 & \\
\hline & Completar sequências & 86 & \\
\hline & Reconhecer ordinais & 87 & \\
\hline \multirow{4}{*}{ Operações } & Juntar, acrescentar e retirar & 82 & \multirow{4}{*}{64} \\
\hline & Completar e comparar & 63 & \\
\hline & Multiplicação & 50 & \\
\hline & Divisão & 60 & \\
\hline \multirow{2}{*}{ Geometria } & Figuras planas & 89 & \multirow{2}{*}{91} \\
\hline & Figuras espaciais & 92 & \\
\hline \multirow{3}{*}{$\begin{array}{l}\text { Grandezas } \\
\text { e medidas }\end{array}$} & Comparar comprimentos & 76 & \multirow{3}{*}{71} \\
\hline & Sistema monetário & 82 & \\
\hline & Leitura de horas & 56 & \\
\hline \multirow{2}{*}{$\begin{array}{l}\text { Tratamento } \\
\text { Informação }\end{array}$} & Tabelas & 74 & \multirow{2}{*}{84} \\
\hline & Gráficos & 87 & \\
\hline
\end{tabular}

Fonte: Elaboração do autor.

Os dados mostram que os rendimentos mais baixos estão associados ao trabalho com as operações e com as grandezas, enquanto as ideias de número, geometria e tratamento da informação apresentam os maiores índices de acertos por parte das crianças. É necessário ressaltar que esses dois últimos blocos de conteúdos são, ainda hoje, os menos trabalhados na escola, enquanto se gasta um tempo considerável com as operações (leia-se algoritmos formais) e cálculo de medidas de grandezas. Câmara (2006) já apontava esse fenômeno em alunos de quinto ano do ensino fundamental, inferindo que a ênfase em procedimentos formais de cálculo os leva a não atribuir sentido às situações, enquanto situações envolvendo conceitos de geometria e leitura de gráficos e tabelas geram 
mais significado para eles, na medida em que são conteúdos presentes em seu cotidiano.

\section{DESCRITOR D2.1}

Os 14 itens desse descritor contemplam as ideias de juntar (2 itens), acrescentar (5 itens) e retirar (7 itens). Os resultados são mostrados na tabela abaixo.

TABELA 2 - Rendimento do descritor D2.1

\begin{tabular}{l|c}
\hline IDEIA & \% DE ACERTO \\
\hline Juntar & 91 \\
\hline Acrescentar & 81 \\
\hline Retirar & 81 \\
\hline
\end{tabular}

Fonte: Elaboração do autor.

Em se tratando de crianças ao final do segundo ano de escolarização, esse resultado não demonstra nenhuma novidade. De fato, a primeira ideia de adição explorada em nossas salas de aula trata da ação de juntar quantidades de elementos de conjuntos para determinar o total de elementos. Apesar de as ações de juntar e acrescentar se referirem à mesma operação, a adição, os procedimentos adotados pelos alunos podem diferenciar em função da estrutura semântica do problema.

Tomemos, por exemplo, a adição $5+3$ e duas situações que envolvam as ações de juntar e acrescentar. No primeiro caso, a determinação do total é feita pela contagem dos elementos com base no primeiro elemento do primeiro conjunto até o último elemento do segundo conjunto $(1,2,3,4,5,6,7,8)$, sendo o último elemento contado o cardinal da união. No caso da ideia de acrescentar, a contagem é realizada com base no último elemento do primeiro conjunto $(5 \ldots 6,7,8)$. Nesse caso, muitas vezes, as crianças fazem a dupla contagem do último elemento do primeiro conjunto $(5,6,78)$, obtendo uma unidade a mais no resultado.

Por outro lado, percebemos diferenças importantes nos rendimentos quando consideramos como variável a presença ou não de uma imagem suporte, como mostra a tabela seguinte. 
TABELA 3 - Rendimento em função da presença de imagem no descritor D2.1

\begin{tabular}{l|c}
\hline SUPORTE & \% DE ACERTO \\
\hline Com imagem & 77 \\
\hline Sem imagem & 87 \\
\hline
\end{tabular}

Fonte: Elaboração do autor.

Observa-se que, nesse caso, a presença da imagem se torna um elemento dificultador. Apesar de merecer estudos mais aprofundados sobre a relação entre presença da imagem e rendimento, a própria característica da prova pode explicar a diferença de rendimento. Partindo do pressuposto de que alunos dessa faixa de escolaridade ainda não estão plenamente alfabetizados, todos os itens são lidos pelo professor e os enunciados e comandos não aparecem no instrumento deles. No caso de problemas que não apresentam imagens no suporte, apenas as alternativas aparecem na prova dos alunos.

Com isso, eles prestam maior atenção à leitura feita pelo professor, ao contrário dos problemas com presença da imagem. Neste caso, os alunos relaxam na atenção, o que os leva, muitas vezes, a simplesmente contar os elementos presentes. Isso aparece de maneira marcante quando analisamos a localização dos dados associados às operações, como mostra a tabela a seguir.

TABELA 4 - Rendimento em função da localização dos dados no descritor D2.1

\begin{tabular}{l|c}
\hline LOCALIZAÇÃO & \% DE ACERTO \\
\hline Apenas na imagem & 72 \\
\hline Na imagem e no texto & 80 \\
\hline
\end{tabular}

Fonte: Elaboração do autor.

Nos itens em que os dados são apresentados somente na imagem, é fornecido um comando do tipo "veja os objetos de Fulano". Nesse caso, os alunos devem realizar previamente a contagem dos elementos para poder realizar a operação. Isso poderia explicar a diferença de oito pontos percentuais entre os dois tipos de itens. 
Nos itens em que os dados aparecem somente na imagem, observamos que aproximadamente um em cada quatro alunos escolhe como alternativa correta aquela que apresenta o número de elementos presentes na imagem. Já quando os dados são lidos pelo professor, o recurso à simples contagem de elementos é utilizado por $14 \%$ dos alunos.

Essefenômeno se vê reforçado quando comparamos o sucesso dos alunos em relação à variável presença (ou não) de imagem como suporte do item, como mostra a tabela abaixo.

TABELA 5 - Rendimento em função da presença de imagem no descritor D2.1

\begin{tabular}{l:c}
\hline VARIÁVEL & \% DE ACERTO \\
\hline Presença de imagem & 77 \\
\hline Ausência de imagem & 87 \\
\hline
\end{tabular}

Fonte: Elaboração do autor.

Também aqui, a forte tendência dos alunos de realizar contagem de elementos da imagem pode explicar a diferença de 10 pontos percentuais no rendimento deles. Essa tendência se vê reforçada na medida em que, por razões técnicas, as questões envolvendo contagem, por serem mais fáceis, aparecem sempre no início dos cadernos de prova. Com isso, ao encontrarem um problema envolvendo números, eles buscam recuperar as estratégias mobilizadas no espaço de tempo imediatamente anterior, ou seja, a contagem de elementos.

Em relação à magnitude dos números envolvidos, encontramos pouca diferença de rendimento nos casos de adição em função do total envolvido no problema, como mostra a tabela seguinte.

TABELA 6 - Rendimento em função da magnitude do total em problemas de adição no descritor D2.1

\begin{tabular}{l|c}
\hline TOTAL & \% DE ACERTO \\
\hline Até 10 & 83 \\
\hline Maior que 10 (até 20) & 86 \\
\hline
\end{tabular}

Fonte: Elaboração do autor. 
Ao contrário do que recomenda o senso comum, os alunos apresentam melhor rendimento, mesmo que por pequena diferença, em problemas envolvendo totais maiores. Entretanto, quando se trata de problemas envolvendo subtração, a diferença cresce bastante, como mostra a tabela a seguir.

TABELA 7 - Rendimento em função da magnitude do minuendo em problemas de subtração no descritor D2.1

\begin{tabular}{l:c}
\hline TOTAL & \% DE ACERTO \\
\hline Até 10 & 86 \\
\hline Maior que 10 (até 20) & 77 \\
\hline
\end{tabular}

Fonte: Elaboração do autor.

Observa-se, nesse caso, que a diferença de rendimento salta para 9 pontos percentuais. É importante destacar que, nos casos em que o minuendo é maior que 10 , seu algarismo das unidades é menor que o algarismo do subtraendo, por exemplo, no caso de 12-3. Considerando a ideia de retirada, a estratégia adequada seria realizar a contagem decrescente $(11,10,9)$. Entretanto, boa parte dos alunos que observamos durante a pré-testagem reconhece a necessidade de realizar uma adição, mas, em vez de realizar a contagem decrescente, eles buscam representar (geralmente escrevendo na mesa) o algoritmo formal da subtração, o que leva ao caso da subtração com recurso ao empréstimo. Isso pode estar associado a questões de ordem didática. De fato, é comum em nossas escolas iniciar o trabalho com as operações com base nos algoritmos formais para, somente depois, utilizá-los na resolução de problemas, que, na verdade, se caracterizam como exercícios de fixação. Segundo Câmara (2008, p. 12),

[...] as consequências de tais escolhas têm se refletido de forma bastante marcante nos resultados de nossos alunos, além de contribuir para o grande índice de evasão em nossas escolas, na medida em que não conseguimos dar significado à matemática que estamos ensinando.

Entretanto, a maior diferença de rendimento em problemas de adição e subtração se encontra em relação à variável contexto do problema, como mostra a tabela abaixo. 
TABELA 8 - Rendimento em função do contexto em problemas do descritor D2.1

\begin{tabular}{l|c}
\hline TOTAL & \% DE ACERTO \\
\hline Contexto familiar & 90 \\
\hline Contexto não familiar & 75 \\
\hline
\end{tabular}

Fonte: Elaboração do autor.

Como contextos familiares categorizamos "lápis”, “crianças” e "animais de estimação". Já na categoria de contextos não-familiares encontramos "conchas", "pratos" e "pinos de boliche”. Isso nos instiga a, em estudos posteriores, buscar identificar em que medida contextos familiares facilitam ao aluno elaborar sentido para o problema e, dessa forma, recorrer a estratégias diferenciadas para resolver os problemas envolvendo operações.

A influência do contexto na resolução de problemas aritméticos precisa ser mais bem investigada. Na pré-testagem dos itens para a Provinha de Matemática, foi colocado um item, unicamente para fins de pesquisa, em que foi apresentada para o aluno uma "conta seca”, ou seja, uma adição apenas em linguagem simbólica, solicitando o resultado. $\mathrm{O}$ índice de acertos nesse item foi de $92 \%$, enquanto a mesma operação em um problema com a ideia de juntar teve apenas $76 \%$ de acertos.

\section{DESCRITOR D2.2}

Esse descritor contemplou 11 itens, sendo 8 itens com a ideia de completar e 3 itens com a ideia de comparar. Na realidade foram pré-testados mais itens de comparação, porém, devido aos resultados bastante baixos, a grande maioria deles foi descartada. Os resultados são mostrados na tabela seguinte.

TABELA 9 - Rendimento por ideia do descritor D2.2

\begin{tabular}{l|c}
\hline IDEIA & \% DE ACERTO \\
\hline Completar & 65 \\
\hline Comparar & 57 \\
\hline
\end{tabular}

Fonte: Elaboração do autor. 
A literatura tem evidenciado que esses tipos de problemas são mais difíceis para os alunos. A dificuldade parece estar, principalmente, na estrutura semântica do problema. De fato, nos problemas de comparação, a expressão “a mais” induz o aluno a considerar o problema como sendo de adição, quando na realidade a operação necessária é de subtração. Isso acontece, principalmente, pela prática frequente em sala de aula de associar palavras-chave às operações aritméticas.

Em relação aos problemas de completar, a presença ou não de imagens como suporte não mostrou diferença significativa de rendimento. Em problemas com imagem, o índice de acertos foi de $65 \%$, enquanto em problemas sem a presença de imagem foi de $64 \%$. Já no caso de problemas com a ideia de comparar, a imagem provoca diferenças importantes no rendimento dos alunos, como mostra a tabela seguinte.

TABELA 10 - Rendimento nos problemas de comparação em função da presença de imagem no descritor D2.2

\begin{tabular}{l|c}
\hline VARIÁVEL & \% DE ACERTO \\
\hline Presença de imagem & 67 \\
\hline Ausência de imagem & 37 \\
\hline
\end{tabular}

Fonte: Elaboração do autor.

No único problema de comparação sem a presença de imagem, quase $30 \%$ dos alunos escolheram como resposta o valor correspondente à soma dos dois valores, confirmando o que apresentamos anteriormente, em que a tendência dos alunos é associar a expressão "a mais" a uma adição.

Nos outros dois problemas, a presença de duas imagens, uma para cada quantidade, pode facilitar a mobilização da estratégia que consiste em estabelecer relações entre os elementos de cada coleção, contando aqueles que não ficaram relacionados. Entretanto, essa estratégia somente foi adotada em um dos problemas, aquele em que os elementos dos dois conjuntos são de mesma natureza, apesar de a magnitude das quantidades envolvidas ser maior que dez.

No outro problema, com valores menores que dez, os elementos dos dois conjuntos são diferentes, apesar de se mostrarem em linha, ao contrário do problema anterior, 
onde os objetos são apresentados de forma desorganizada. Podemos observar que, no caso em que os objetos são diferentes, mais da metade dos alunos escolheu como estratégia contar os elementos de uma das duas coleções, sem estabelecer relações entre eles.

Da mesma forma que no descritor anterior, a localização dos dados do problema afetou o rendimento dos alunos, como mostra a tabela a seguir.

TABELA 11 - Rendimento em função da localização dos dados no descritor D2.2

\begin{tabular}{l|c}
\hline LOCALIZAÇÃO & \% DE ACERTO \\
\hline Apenas na imagem & 58 \\
\hline Na imagem e no texto & 72 \\
\hline
\end{tabular}

Fonte: Elaboração do autor.

Também aqui, nos problemas em que os alunos devem realizar a contagem dos elementos para realizar a operação, verificamos maior tendência de dar como resposta o número de elementos de um dos dois conjuntos (23\%) do que nos problemas em que os dados aparecem no enunciado (18\%).

Em relação à magnitude dos números envolvidos, o rendimento dos alunos é melhor em problemas com números maiores que dez, como mostra a tabela abaixo.

TABELA 12 - Rendimento em função da magnitude dos números no descritor D2.2

\begin{tabular}{l:c}
\hline MAGNITUDE & \% DE ACERTO \\
\hline Até 10 & 58 \\
\hline Maiores que 10 & 72 \\
\hline
\end{tabular}

Fonte: Elaboração do autor.

Nos problemas com a ideia de comparar em que o minuendo é maior que dez e o subtraendo é menor que dez, encontramos uma variação importante de rendimento em função dos números envolvidos. Temos três problemas em que o subtraendo é menor que o valor do algarismo das unidades do minuendo (17 -4, por exemplo) e dois problemas em que o 
subtraendo é maior que o algarismo das unidades do minuendo (13-8, por exemplo).

No primeiro caso, o índice de sucesso é de $71 \%$, contra $58 \%$ no segundo caso, embora nos dois problemas a ideia de base seja de completar uma quantidade para atingir outra. Entretanto, as observações feitas durante a aplicação do pré-teste mostram que poucos alunos mobilizam efetivamente a ideia de completar em problemas dessa natureza. Eles reconhecem a operação de subtração como aquela que resolve o problema, mas, por conta de determinadas regras estabelecidas implicitamente em nossas salas de aula, buscam utilizar o algoritmo formal para realizar a operação. Visto que no caso de subtraendo maior que as unidades do minuendo é exigido o recurso ao empréstimo, na realização do algoritmo formal, o índice de acertos decresce de maneira importante.

\section{CONSIDERAÇÕES FINAIS}

Nosso objetivo neste trabalho foi de identificar como as variáveis envolvidas nos problemas de estrutura aditiva afetam o sucesso dos alunos em situação de avaliação, caso da Provinha Brasil de Matemática. Acreditamos que a utilização dos resultados da pré-testagem dos itens para a Provinha, com a participação de 12 mil alunos de diferentes partes do país, poderia nos oferecer resultados com certa estabilidade, ou seja, que permitam uma certa generalização dos resultados.

A análise dos dados fornecidos pelo tratamento estatístico, associada às observações que pudemos realizar durante a pré-testagem, nos mostra que diferenças devariáveis nos problemas, mesmo que sutis, afetam bastante o sucesso dos alunos nos itens. Em particular, tomamos como foco o contexto do problema, a presença ou ausência de imagem no suporte, a magnitude dos valores envolvidos e a localização dos dados numéricos.

Acreditamos que, com esses resultados, torna-se necessário realizar investigações com metodologias adequadas (como entrevistas, por exemplo), para identificar que estratégias os alunos efetivamente mobilizam em função dos valores de cada uma dessas variáveis nos problemas. 


\section{REFERÊNCIAS}

ARAUJO, Abraão; CÂMARA, Marcelo. Desempenho dos alunos do programa projovem no exame de avaliação externa no caso de áreas e volumes. Bolema: Boletim de Educação Matemática, Rio Claro, n. 33, 2009.

BRASIL. Ministério da Educação. Parâmetros Curriculares Nacionais de Matemática: ensino de primeira a quarta série. Brasília: MEC/SEF, 1997.

CÂMARA, Marcelo. Avaliar com os pés no chão... da classe de matemática. In: CARVALHO, Maria Helena; UYTDENBROEK, Xavier (Org.). Avaliar com os pés no chão da escola: reconstruindo a prática pedagógica no ensino fundamental. Recife: UFPE, 2000.

Professor, afinal, quem inventou essa tal de matemática? In: ENCONTRO NACIONAL DE DIDÁTICA E PRÁTICA DE ENSINO, 3., 2006. Anais... Recife: Bagaço, 2006.

A Matemática na sala de aula, ou como transformar singelas vaquinhas em diabólicos monômios. In: SIMPÓSIO INTERNACIONAL DE PESQUISA EM EDUCAÇÃO MATEMÁTICA, 2. Anais... Recife: UFRPE, 2008.

Nossos alunos não sabem somar? O que (não) dizem os resultados da avaliação em larga escala de Pernambuco. In: SEMINÁRIO INTERNACIONAL DE PESQUISA EM EDUCAÇÃO MATEMÁTICA, 4., 2009. Anais... Taguatinga: SBEM, 2009.

CÂMARA, Marcelo; MELO, Diógenes. Por quem? Por cem! o que dizem os alunos do Projovem sobre porcentagem no exame nacional externo. In: REUNIÃO ANUAL DA ASSOCIAÇÃO BRASILEIRA DE AVALIAÇÃO EDUCACIONAL, 5. Anais... Salvador: Abave, 2009.

VERGNAUD, Gérard. A Classification of cognitive tasks and operations of thought involved in addition and subtraction problems. In: CARPENTER, Mose; ROMBERG, Thomas A. (Org.). Addition and subtraction: a cognitive perspective. New York: Academic, 1982.

La Théorie des champs conceptuels. Recherches en Didactique des Mathématiques, Grenoble, n. 10, 1990. 


\section{MARCELO CÂMARA}

Professor do Programa de Pós-graduação em Educação Matemática e Tecnológica da Universidade Federal de Pernambuco (Edumatec/UFPE). Professor do Programa de Pós-Graduação Profissional em Gestão e Avaliação da Educação Pública (PPGP) do Centro de Políticas Públicas e Avaliação da Educação (CAEd) da Universidade Federal de Juiz de Fora (UFJF)

marcelocamaraufpe@yahoo.com.br 